ICAMS 2016 $-6^{\text {th }}$ International Conference on Advanced Materials and Systems

\title{
COLLAGEN NETWORKS OBTAINED BY LEATHER INDUSTRY BY-
} PRODUCTS

\author{
BOGDAN CURSARU ${ }^{1,2}$, MIHAELA-DOINA NICULESCU ${ }^{1}$, MARIANA DANIELA \\ BERECHET $^{1}$, MIHAI RADU ${ }^{3}$, MĂDĂLINA CAMELIA IGNAT ${ }^{1}$, LAURA-MĂDĂLINA \\ POPESCU $^{4}$, IOAN ALBERT TUDOR ${ }^{4}$ \\ ${ }^{1}$ INCDTP - Division Leather and Footwear Research Institute, 93 Ion Minulescu st., District 3, \\ Bucharest,031215, Romania,bogdancursaru@yahoo.com \\ ${ }^{2}$ National Research and Development Institute for Chemistry and Petrochemistry ICECHIM, \\ Splaiul Independentei no. 202, Bucharest, 060021, Romania \\ ${ }^{3}$ University POLITEHNICA of Bucharest, 1-7 Gheorghe Polizu St., Bucharest, 011061, Romania \\ ${ }^{4}$ National R\&D Institute for Non-ferrous and Rare Metals (INCDMNR-IMNR), 102 Biruintei \\ Blvd., Pantelimon, Ilfov County, 077145, Romania
}

\begin{abstract}
Chemically cross-linked collagen polymeric networks with different structures and properties were obtained from collagen hydrolysates and glutaraldehyde and were designed for potential applications in bioeconomy. The swelling behavior of these networks was tested in distilled water at constant temperature and the equilibrium swelling degree was calculated. The influence of different cross-linking parameters used to tailor the structure and properties of these networks (collagen-glutaraldehyde ratio and the aminic nitrogen content of the collagen hydrolysate) upon the collagen networks was investigated by FTIR spectroscopy, thermal analysis, swelling tests and contact angle measurements. Different amounts of glycerol were added in order to further improve the general properties of the collagen networks. The general properties of these materials leaded to the conclusion that they could be used for potential applications in bioeconomy.
\end{abstract}

Keywords: Collagen hydrolysate, cross-linking, bioeconomy

\section{INTRODUCTION}

Hydrogels are three-dimensional cross-linked polymeric networks which exhibit the ability to swell and retain large volumes of water in their structure, without dissolution. Their resistance to dissolution is given by the cross-links between network chains. Based on the type of these cross-links, hydrogels can be classified in two categories: chemical or permanent hydrogels, which exhibit chemical junctions between polymeric chains, and physical hydrogels which presents temporary junctions that arise from weak physical interactions, such as hydrogen bonds, chain entanglements, and ionic or hydrophobic interactions. Hydrogels can be also divided in two main categories, based on the nature of the polymeric chains: natural, synthetic polymers, or combination of these two categories. Natural hydrogels are made up from polymers like collagen, gelatin, starch, agarose, and alginate. Synthetic hydrogels are obtained from chemically synthesized polymers (Ahmed, 2015).

Collagen is one of the most important and abundant proteins present in animals and humans bodies and plays a key role in the extracellular matrix and in many tissues like skin, bone, cartilage, tendons (Niculescu and Gaidau, 2014; Zhang et al., 2014; Fiorani et al., 2014). Collagen is currently extracted from this tissues using chemical-enzymatic or chemical methods (Niculescu and Gaidau, 2014; Fiorani et al. 2014). Collagen hydrolysate is a polypeptide compound made by further hydrolysis of collagen, which displays the ability to form thin films on different surfaces, has a hydrophilic character and is not dangerous for human health. Due to these remarcable properties, it has been used in various applications, including as a natural fertilizer in agriculture due to the release of free amino acids with a considerable nitrogen content (Niculescu and Gaidau, 2014; Pei et al., 2013). Other applications of collagen hydrolyates are in regenerative medicine, in pharmaceutics, in 
cosmetics, as a food additive and as a biodegradable packing material (Pei et al., 2013). Unlike gelatin, collagen hydrolyates displays a lower molecular weight and better biodegradability and absorbency (Pei et al., 2013). The mechanical and thermal stability of the collagen physical hydrogels can be further improved by employing various cross-linking agents, such as: formaldehyde (Zhang et al., 2014; Tan et al., 2015), glutaraldehyde (Zhang et al., 2014; Osborne et al., 1997; Tan et al., 2015; Rault et al., 1996), glycosaminoglycan (Osborne et al., 1997), chondroitin-6-sulphate (Osborne et al., 1997), 1-ethyl-3-(3dimethylaminopropyl) carbodiimide (Osborne et al., 1997; Sionkowska et al., 2010, Rault et al., 1996), 1,1-carbonyldiimidazole (Osborne et al., 1997), hexamethylene diisocyanate (Zhang et al., 2014; Rault et al., 1996), cyanamide (Rault et al., 1996), Nhydroxysuccinimide (Sionkowska et al., 2010; Tan et al., 2015), diepoxypropylether (Sionkowska et al., 2010), hydrazine (Rault et al., 1996), diphenylphosphorylazide (Rault et al., 1996), dialdehyde carboxymethyl cellulose (Tan et al., 2015) and genipin (Zhang et al., 2014; Biswal et al., 2016). Among these cross-linkers, glutaraldehyde was widely used for the collagen/gelatine hydrogels preparation due to its high reactivity, high water solubility and low cost. The reaction mechanism of collagen cross-linking by glutaraldehyde consists of the chemical reaction between the free amino functional groups of different amino acids from collagen and the carbonyl functional group from glutaraldehyde, with the formation of a Schiff base type structure (Tian et al., 2016).

The collagen hydrogels display high biocompatibility and biodegradability, whereas their mechanical and thermal properties were relative low (Nistor et al., 2015). In order to overcome this disadvantages, the collagen hydrogels were additivated with various compounds, such as cellulose (Pei et al., 2013), glycerol or poly(ethylene glycols) of different molecular weights (Langmaier et al., 2008).

The aim of this research is to obtain new 3-D structures by reusing collagen byproducts from the leather industry. Moreover, the target of this research is to transform low-economical value by-products from leather industry into value-added products for bioeconomy, following the concept of economical and environmental sustainability.

\section{EXPERIMENTAL}

The bovine collagen hydrolysates were obtained according the procedure described (Niculescu and Gaidau, 2014), and their characteristics are presented in Table 1.

Table 1. Physico-chemical characteristics for the bovine collagen hydrolysates employed for the 3-D collagen structures synthesis

\begin{tabular}{llrrl}
\hline No. & Characteristics, MU & HCN2 & HCN3 & Standard \\
\hline 1 & Dry substance, \% & 9.80 & 9.59 & SR EN ISO 4684:2006 \\
2 & Total ash, \% & 5.92 & 7.09 & SR EN ISO 4047:2002 \\
3 & Total nitrogen, \% & 16.52 & 16.79 & SR EN ISO 5397:1996 \\
4 & Free amino nitrogen, \% & 1.84 & 1.55 & ICPI internal standard \\
5 & Organic substance, \% & 92.90 & 94.36 & SR EN ISO 5397:1996 \\
6 & Calcium oxide, \% & 4.90 & 3.43 & ICPI internal standard \\
7 & Chromium oxide, \% & \multicolumn{2}{c}{ Values below } & SR EN ISO 5398-1:2008 \\
& & the detection limit (0.3\%) & \\
8 & pH & 8.50 & 8.77 & STAS 8619/3:1990 \\
\hline
\end{tabular}

The bovine collagen hydrolysate HCN3 was employed for the formation of HCG-5 and HCG-9 hydrogels, whereas HCG-11 was obtained from the HCN2 collagen hydrolysate 
ICAMS $2016-6^{\text {th }}$ International Conference on Advanced Materials and Systems

(which displays a higher free amino $\mathrm{N}_{2}$ content). In all cases the crosslinking agent was a $25 \%$ aqueous solution of glutaraldehyde. The HCG-9 and HCG-11 hydrogels were additivated with $25 \%$ and $2.5 \%$ glycerol, respectively, whereas HCG-5 was not additivated.

The FTIR spectra of the collagen xerogels were recorded in the transmission mode (FTIR-ATR) on a ABB MB3000 spectrometer. The IR spectra was recorded in the wavenumbers range of $4000-550 \mathrm{~cm}^{-1}$ and consists of 64 scans at $4 \mathrm{~cm}^{-1}$ spectral resolution. The DSC measurements of the collagen xerogels were performed on a Netzsch DSC200 F3 Maia thermal analysis system. The measuring conditions were the following: temperature range: $-20-400^{\circ} \mathrm{C}$, the heating rate was $10^{\circ} \mathrm{C} / \mathrm{min}$, and the Argon flow rate was $1 \mathrm{Lh}^{-1}$. The contact angle of a water droplet on the xerogel surface was measured with the VCA Optima XE device. The surface energy was determined by the means of the VCA Optima XE device software. For the swelling tests, the xerogel thickness was measured using a micrometer.

\section{RESULTS AND DISCUSSION}

The free amino nitrogen content provides valuable information for the free amino groups available for the cross-linking reaction with glutaraldehyde. The HCN2 hydrolysate had a free amino nitrogen content of $1.84 \%$, whereas HCN3 had $1.55 \%$. After $\mathrm{pH}$ adjustment at the value of 7.4, the reaction mixture (collagen hydrolysate, glutaraldehyde and glycerol) was very well stirred and injected through a $2.5 \mathrm{~mm}$ thick circular rubber gasket between two parallel glass square plates (as described in Cursaru et al., 2008), as one can see in Figure 1A. This device was kept in an oven at $40^{\circ} \mathrm{C}$ for 1 hour. At the end of the time, the device was opened and the hydrogel circular plate was cut into $10 \mathrm{~mm}$ diameter disks. These disks were after immersed into a determined volume of distilled water (considered in excess), and kept 6 days at room temperature with water changing every day, in order to remove the unreacted species. Figure 1B presents the increased volume of the collagen hydrogel at equilibrium swelling, as compare with the initial dry disk (xerogel).
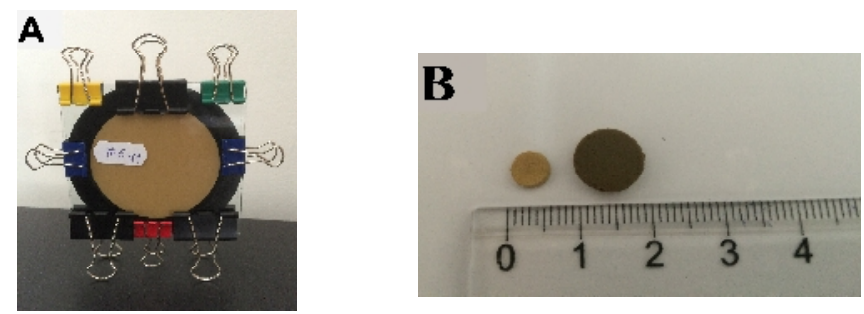

Figure 1. The device employed in the synthesis of the collagen networks (A); Hydrogel disks at the equilibrium swelling at $28^{\circ} \mathrm{C}$ (B)

Figure 2 comparatively presents FT-IR spectra of collagen hydrolysate (HCN3) and of the corresponding hydrogel (HCG-5). In these spectra, only HCG-5 displayed the two characteristic peaks of a Schiff base: at $3325 \mathrm{~cm}^{-1}$ (assigned to stretching vibration of $\mathrm{N}-\mathrm{H}$ group) and at $1670 \mathrm{~cm}^{-1}$ (assigned to carbonyl groups). The presence of these peaks demonstrates the occurrence of the cross-linking reaction between collagen hydrolysate and glutaraldehyde. Both the collagen hydrolysate (HCN3) and its corresponding collagen network (HCG-5) displayed the following peaks, respectively: 
$v_{\mathrm{O}-\mathrm{H}}$ stretching vibration at 3069 and $3204 \mathrm{~cm}^{-1}$; asymmetric stretching vibrations of $\mathrm{CH}_{2}$ group at $2947-2874 \mathrm{~cm}^{-1}$ and $2945-2872 \mathrm{~cm}^{-1}$; characteristic bands of amide I (1637-1657 $\mathrm{cm}^{-1}$ in the case of HCN3 and $1655 \mathrm{~cm}^{-1}$ for HCG-5) due to $v_{\mathrm{C}=0}$; amide II (at 1540 and $1560 \mathrm{~cm}^{-1}$ ) due to $v_{\mathrm{C}-\mathrm{N}}$ and $\delta_{\mathrm{N}-\mathrm{H}}$; amide III at $1248 \mathrm{~cm}^{-1}$ for HCN3 and $1240 \mathrm{~cm}^{-1}$ in the case of HCG5 (due to $v_{\mathrm{C}-\mathrm{N}}$ and $\delta_{\mathrm{N}-\mathrm{H}}$ ).

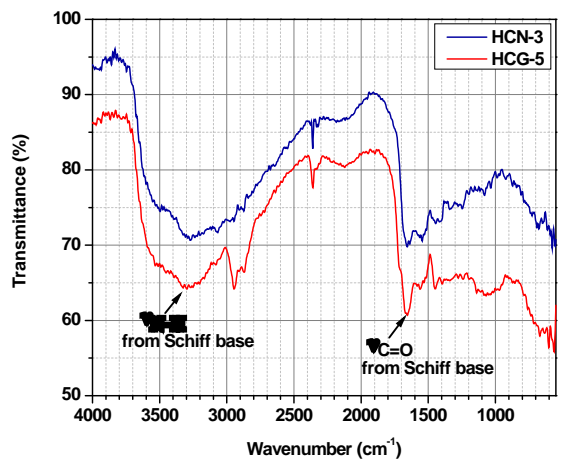

Figure 2. FT-IR spectra of collagen hydrolysate (HCN3) and of the corresponding hydrogel (HCG-5)

Swelling studies were done, in order to comparatively analyse the swelling behavior of the 3-D collagen networks. The swelling degree was determined by immersing a weighted xerogel disk $\left(\mathrm{W}_{0}\right)$ (with a diameter around $6 \mathrm{~mm}$ and a thickness in the range of 1.25-1.5 mm) into a determined volume of distilled water at a constant temperature $\left(28^{\circ} \mathrm{C}\right)$. The disks were extracted from the water at certain time intervals, superficially wiped with filter paper, weighted $\left(\mathrm{W}_{\mathrm{t}}\right)$ and sank again. The swelling experiments were carried out until the swelling degree of the hydrogel reached a constant value, corresponding to the equilibrium swelling degree. The swelling degree was determined according to equation (1) (Cursaru et al., 2008):

$\mathrm{SD}=\left(\mathrm{W}_{\mathrm{t}}-\mathrm{W}_{0}\right) / \mathrm{W}_{0}$

Evolution of the swelling degree in time for the HCG-5, HCG-9 and HCG-11 hydrogels is presented in Figure 3.

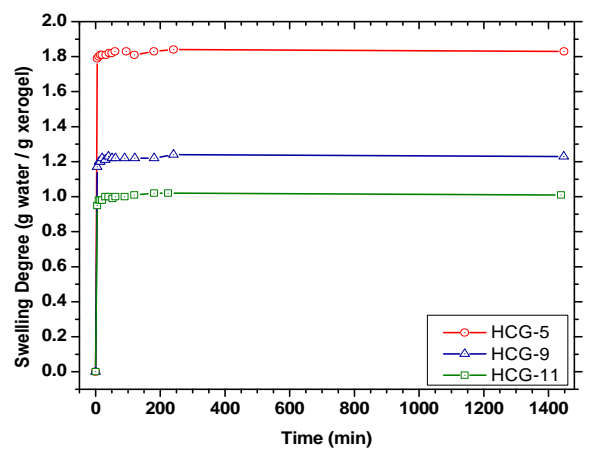

Figure 3. Evolution of the swelling degree in time for the HCG-5, HCG-9 and HCG-11 hydrogels 
ICAMS $2016-6^{\text {th }}$ International Conference on Advanced Materials and Systems

The equilibrium swelling degree (ESD) values were 1.83 for HCG-5, 1.23 in the case of HCG-9 and 1.02 corresponding to HCG-11.

Both the contact angle of a water droplet on a xerogel surface and the calculated surface energy are depicted in Figure 4, and together reveals the hydrophilic character of the hydrogels analysed.
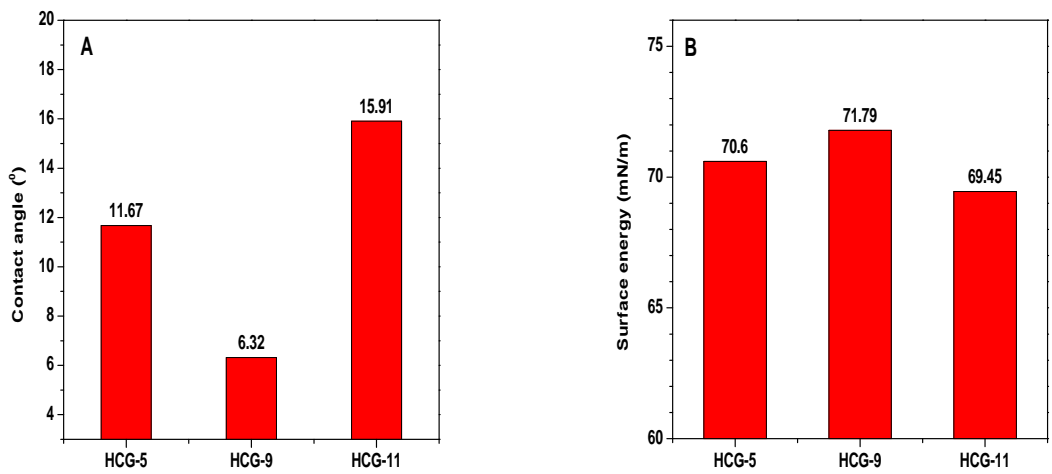

Figure 4. Contact angle of collagen networks (A) and the calculated surface energy (B) on HCG-5, HCG-9 and HCG-11 disks surface

From DSC curves depicted in Figure 5, one can observe a superior thermal stability of the xerogel (HCG-11) compared with the precursor hydrolysate (HCN2). This could be due to the cross-links present in the hydrogel network. Moreover, thermal degradation occurs in only two steps (at $180^{\circ}$ and $333.8^{\circ} \mathrm{C}$ ), comparing to collagen hydrolysate precursor, where more endothermic peaks can be observed.
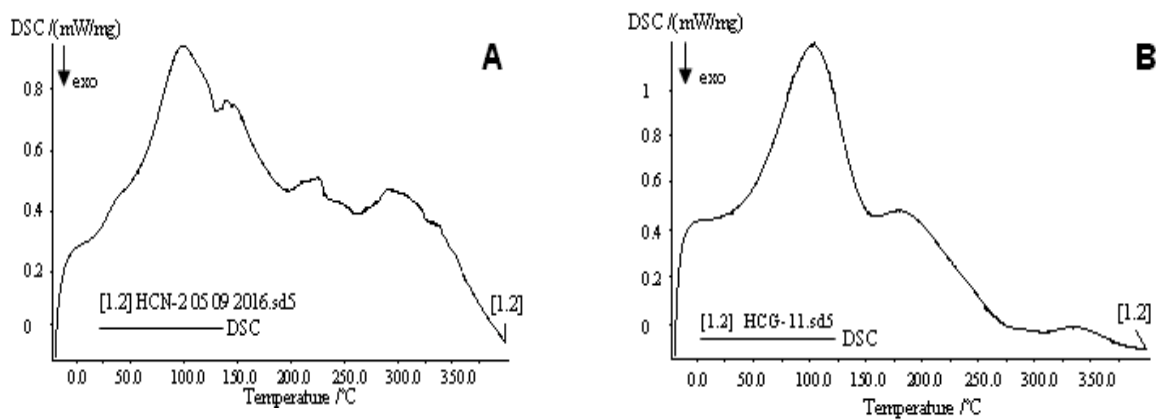

Figure 5. DSC curve for HCN2 hydrolysate and for HCG-11 xerogel (plasticized with $2.5 \%$ glycerol)

\section{CONCLUSIONS}

Some new collagen 3-D networks with potential applications in bioeconomy were obtained from two different bovine collagen hydrolysates and glutaraldehyde.

The FT-IR spectra revealed the characteristic peak of a Schiff-base compound only on collagen hydrogels spectra, which demonstrated the occurrence of cross-linking 
reaction between free amino groups present in collagen hydrolysates and the carbonyl function of glutaraldehyde.

The DSC thermograms revealed higher thermal stability in the case of the collagen hydrogels additivated with glycerol in comparison with the precursor collagen hydrolysate.

The contact angle determination demonstrated the hydrophilic character of the collagen hydrogels.

The HCG-5 hydrogel exhibited a higher swelling degree in comparison with the two others hydrogels, which contains glycerol.

\section{Acknowledgement}

The authors gratefully acknowledge the financial support of National Core Programme INOVA-TEX-PEL, project no. PN.16.34.01.07.

\section{REFERENCES}

Ahmed, E.M. (2015), "Hydrogel: Preparation, characterization, and applications: A review", Journal of Advanced Research, 6, 105-121.

Biswal, D., Anupriya, B., Uvanesh, K., Anis, A., Banerjee, I. and Pal, K. (2016), "Effect of mechanical and electrical behavior of gelatin hydrogels on drug release and cell proliferation", Journal of the Mechanical Behavior of the Biomedical Materials, 53, 174-186.

Cursaru, B., Stănescu, P. and Teodorescu, M. (2008), "Compression properties of hydrogels synthesized from diepoxy-terminated poly(ethylene glycol)s and aliphatic polyamines", Materiale Plastice, 45(4), 314-319.

Fiorani, A., Gualandi, C., Panseri, S., Montesi, M., Marcacci, M., Focarete, M.L. and Bigi, A. (2014), "Comparative performance of collagen nanofibers electrospun from different solvents and stabilized by different crosslinkers", Journal of Materials Science: Materials in Medicine, 25, 2313-2321.

Langmaier, F., Mokrejs, P., Kolomaznik, K., and Mladek, M. (2008) "Plasticizing collagen hydrolysate with glycerol and low-molecular weight poly(ethylene glycols)", Thermochimica Acta, 469, 52-58.

Niculescu, M.-D. and Gaidau, C. (2014), "New collagen extracts conditioned for applications in crop protection against pests", Revista de Chimie (Bucharest), 1457-1461.

Nistor, M.T., Vasile, C. and Chiriac, A.P. (2015), "Hybrid collagen-based hydrogels with embedded montmorillonite nanoparticles", Materials Science and Engineering, C53, 212-221.

Osborne, C.S., Reid, W.H. and Grant, M. H. (1997), "Investigation into cell growth on collagen/chondroitin6-sulphate gels: the effect of crosslinking agents and diamines", Journal of Materials Science: Materials in Medicine, 8, 179-184.

Pei, Y., Yang, J., Liu, P., Xu, M., Zhang, X. and Zhang, L. (2013), "Fabrication, properties and bioapplications of cellulose/collagen hydrolysate composite films", Carbohydrate Polymers, 92, 1752 1760 .

Rault, I., Frei, V. and Herbage, D. (1996), "Evaluation of different chemical methods for cross-linking collagen gel, films and sponges", Journal of Materials Science: Materials in Medicine, 7, 215-221.

Sionkowska, A., Skopinska-Wisniewska, J., Gawron, M., Kozlowska, J. and Planecka, A. (2010), "Chemical and thermal cross-linking of collagen and elastin hydrolysates", International Journal of Biological Macromolecules, 47, 570-577.

Tan, H., Wu, B., Li, C., Mu, C., Li, H. and Lin, W. (2015), "Collagen cryogel cross-linked by naturally derivated dialdehyde carboxymethyl cellulose", Carbohydrate Polymers, 129, 17-24.

Tian, Z., Liu, W. and Li, G. (2016), "The microstructure and stability of collagen hydrogel cross-linked by glutaraldehyde", Polymer Degradation and Stability, 130, 264-270

Zhang, X., Chen, X., Yang, T., Zhang, N., Dong, L., Ma, S., Liu, X., Zhou, M. and Li, B. (2014), "The effects of different crossing - linking conditions of genipin on type I collagen scaffolds: an in vitro evaluation", Cell Tissue Bank, 15, 531-541. 